\begin{abstract}
Demand Driven Acquisitions (DDA) of electronic books (ebooks) has been a favored method of acquisitions in academic libraries for a number of years. Despite careful consideration and planning ahead of implementation, snags and hiccups may present in the acquisitions and cataloging workflow while executing a DDA pilot. Technical Services must remain attentive, collaborative, and flexible in order to redress known issues and anticipate potential ones. DDA is ultimately a worthwhile endeavor that has the potential to replace traditional bibliographer ebook title selection, particularly when the long-term integrity of a collections budget is in question.
\end{abstract}

\title{
Evolving Technical Services Workflows in a Demand-Driven Acquisitions Pilot
}

\section{Introduction}

Collection development selectors have long sought ways to more accurately provide users with the right information and required resources, precisely at their point of need. ${ }^{1}$ In tandem with these efforts, academic libraries have been innovating ways to maximize the impact of their collection development dollars, particularly when collection budgets level off over time - or, when adjusted for inflation, decline. Exacerbating the need to make each dollar count is the perceived lack of available funds for monograph title selection, as precious resource dollars are often tied up in large, ongoing subscription-based electronic journal packages, databases, and other similar "Big Deals," ${ }^{2}$ and rainy-day funds or emergency budget augmentations are exhausted. And finally, compounding this austere state of affairs, traditional print and electronic resource subscriptions can be expected to increase anywhere

\footnotetext{
${ }^{1}$ Rebecca Schroeder and Tom Wright, "Electronic Books: A Call for Effective Business Models," New Library World 12, no. 5/6 (2011): 217, doi: 10.1108/03074801111136257.

2 Rebecca Pool, “Big-Deal Packages Squeeze Recession-Hit Libraries," Research Information 48 (2010),

http://www.researchinformation.info/features/feature.php?feature_id=269.
} 
from $5 \%$ to $7 \%$ in a given year, ${ }^{3}$ forcing selectors to further cut back on remaining areas like standing order serials and monographs acquisitions. Thus, some libraries have turned to Demand-Driven Acquisitions (DDA) as a way to stretch their budgets and maximize their dwindling book purchase allocations, while meeting the broadest possible range of user needs. In 2011, the Cal Poly Pomona (CPP) University Library set out to pilot a DDA program.

\section{DDA Overview}

DDA, also commonly referred to as Patron-Driven Acquisitions (PDA), thundered onto the academic library scene in 2011 with Rick Andersen's prediction of a user-selected, point-of-need, fractionally distilled collection of the future. ${ }^{4}$ It is a purchasing model that utilizes user behavior to drive book acquisitions and purchase decisions. There exist different flavors of DDA, which offer selectors the flexibility to intervene (or not) in the acquisitions process, to state a preference for electronic and/or print formats, and to determine the type of resource use that will ultimately trigger a purchase of a given title. Under the most commonly utilized model, MARC records for ebook titles not actually owned by the host library are loaded into the online catalog for patron discovery. ${ }^{5}$ As users interact with the electronic texts, a short-term loan (STL), or even outright purchase, of the text may be triggered. Libraries are able to set the threshold for what constitutes a "use" for STL to be triggered and a cost incurred, including the length of time spent looking at the text, the number of pages viewed, downloads, and printing activity. Libraries may also set the number of times a STL will occur before a purchase is triggered, and selectors may even choose to intervene in the process and actively select the title in advance of any user triggers. Lest users exhaust precious resources in their pursuit of popular reading, expensive textbooks, and other works generally undesired by academic libraries, selectors can fine-tool

\footnotetext{
${ }^{3}$ Serial Price Projections for 2015. Ipswich, MA: Ebsco, 2014. Accessed September 1, 2015, http://www.ebscohost.com/promoMaterials/Serials_Price_Projections_for_2015.pdf.

${ }^{4}$ Rick Anderson, "Collections 2021: The Future of the Library Collection Is Not a Collection," Serials 24, no. 3, (2011): 211-215. http://dx.doi.org/10.1629/24211 ${ }^{5}$ Dee Ann Allison, The Patron-Driven Library: A Practical Guide for Managing Collections and Services in the Digital Age (Oxford: Chandos Publishing, 2013), 92.
} 
a vendor approval profile in keeping with their usual acquisitions policies, and thus limit the pool of discovery records to only those works that would normally be deemed suitable for purchase. Ultimately, user needs are driving purchased content, rather than selectors attempting to predict what resources might be used, and libraries are realizing substantial cost savings over traditional title selection. ${ }^{6}$

\section{The Case for DDA at Cal Poly Pomona}

Several factors drove the decision to trial DDA at CPP. A windfall of extra state lottery funds late in the fiscal year needed to be encumbered, and quickly. Workforce attrition due to retirements and a sustained hiring freeze had left fewer librarians to function as subject liaisons for the university's numerous colleges and departments, and thus significantly increased the purchasing burden on selectors as they found themselves responsible for a new area (or two, or three). Lastly, years of CPP annual reports reflected declining print circulation, soaring online usage statistics, and increased interlibrary loan requests, indicating that the library's print collection was perhaps falling short in meeting diverse user needs.

It should be noted that in order for a DDA pilot to be seriously considered, the Collection Development Team first needed reassurance that a "books" budget would be preserved into the future. For some, concern lingered that a dedicated books budget would suffer with the adoption of yet another online purchasing model, and that favoring electronic format over print was somehow contributing to the overall attrition of the collections budget. However, quite the opposite would be true - the implementation of a DDA program would allow the Collection Development Team to protect and preserve the books budget by securing a large amount of funds within a DDA deposit account, thus safeguarding those funds from appropriation by campus administration. Furthermore, print had not been the bread and butter of the library's serials and periodicals acquisitions since the 2004/05 fiscal

\footnotetext{
${ }^{6}$ Doug Way and Julie Garrison, "Financial Implications of Demand-Driven Acquisitions: A Case Study of the Value of Short-Term Loans," in Patron Driven Acquisitions: History and Best Practices, ed. David A. Swords (Berlin: De Gruyter Saur, 2011), 149-150.
} 
year, when spending on electronic formats surpassed print by over $\$ 10,000$. Though an in-house bindery had been planned for in the library's 2006-08 renovation and addition, it had never actually been built. And all the while, monograph ebook expenditures were increasing and would soon follow serials over the tipping point, outpacing print by nearly $\$ 32,000$ in $2012 / 13$. The reality was that users and selectors alike showed a strong preference for online format for a variety of resources in most disciplines, and that usage of electronic material (and even print) would soon skyrocket with improvements to library resource discovery, vis-à-vis library catalog wrappers like Encore Synergy and Xerxes.

Finally, CPP felt that DDA had achieved a level of approbation which made the undertaking of a pilot feasible. Publications detailing successful DDA projects like the Orbis-Cascade Alliance Pilot redressed concerns arising from previous DDA attempts by other institutions; most notably, that the appropriate number of short-term loans, combined with a carefully crafted approval profile and diligent, collaborative oversight between member libraries and vendors at every step of the process, could result in a viable and sustainable acquisitions model into the future. ${ }^{7}$ These favorable reports, combined with attendance of informational sessions at the 2011 ALA Midwinter Meeting and the outreach efforts of book vendor YBP and ebook provider ebrary, led the librarians of CPP to pilot a DDA project.

\section{Planning, Philosophical Considerations, and Implementation}

The Collection Development Team in April of 2011, with support from the Library Dean, collectively agreed to officially embark upon a DDA pilot. The Collection Development Manager and a team from Bibliographic Access Services (BAS) then worked in close collaboration with YBP to flesh out the details of the program, and develop an acquisition and cataloging workflow. A variety of options

\footnotetext{
7 Jill Emery and Bonnie Parks, "The Demand Driven Acquisitions Pilot by the Orbis Cascade Alliance: An Interview with Members of the Demand Driven Acquisitions Implementation Team," Serials Review 38 (2012): 136, doi: 10.1080/00987913.2012.10765440.
} 
were explored in regards to formats, ebook platforms, number of STLs, the approval profile, cataloging services, and the amount of funds to be earmarked for the pilot.

In keeping with CPP's preference for electronic format, ebooks were chosen as the sole acquisition format for DDA. Ebooks provided distinct advantages over print, most notably in the reduction of receiving and processing physical items, and had a net neutral impact on the Monographs Unit's workload (the additional time spent handling DDA was offset by the simultaneous decline in print acquisitions). Ebrary was selected as the sole ebook aggregator for the pilot for several reasons: a subscription to their platform was already held for the IT \& Computing ebook collection and individually selected ebook purchases; STL and purchase pricing was more straightforward than the complicated non-linear lending model offered by EBL (which has since been acquired by ebrary's parent company, ProQuest); the administration of the DDA account within ebrary's admin site was easy and intuitive to learn and use; and the library's constituency was already familiar with the ebrary platform.

Next, the library chose to partner with vendor YBP for the execution of DDA. YBP's ordering interface, GOBI 3, provided advantages over doing DDA directly with an ebook aggregator. One of the primary benefits of GOBI 3 was the ability of all selectors to log in and make adjustments to DDA discovery content, removing and adding titles as they saw fit without having to pass requests through a sole DDA administrator. Moreover, titles that would be included in DDA discovery could be modeled after pre-existing print approval plans, greatly reducing the amount of work that would go into creating a discovery profile from scratch. GOBI 3 also reflected past purchasing activity, allowing account administrators and selectors to avoid duplication of content in print and electronic formats. Lastly, YBP provided incentive in the form of discounts for academic buyers.

An approval profile was developed based on the existing print approval plan on file with YBP, and further refined to eliminate popular content-level titles, textbooks, self-help works, and anything with a list price over $\$ 200$. It was decided that 2 STLs (each loan instance lasting one day) would be 
allowed, with a purchase being triggered upon the $3^{\text {rd }}$ use of a title. Most publishers in the profile would charge $10 \%$ of list price for each STL, which was to be paid in addition to (not credited towards) the list price of the work upon the triggered purchase. As it was unknown how much DDA might be utilized, 2 STLs (generally 20\%) seemed a reasonable amount of money to expend beyond list price for the lease of a title, should that title prove to be popular. It was also decided that single-user electronic copies were preferred (called Single-User Purchase Option, or SUPO) over the Multiple-User Purchase Option (MUPO) due to their lower cost, and with the safeguard that selectors had the option to intervene and upgrade SUPO to MUPO if high demand for a given title was observed.

YBP was also advantageous in that it provided various levels of ebook cataloging services, from standard vendor-created bibliographic MARC records, to records enriched with book summary and table of contents metadata. At the time, CPP utilized the Encore Synergy discovery layer from Innovative Interfaces, Inc., and so felt that enough quality metadata was provided in the ebrary-supplied vendor records for effective discoverability and recall by users. Thus, they opted for the minimum level of record enhancement from YBP: choosing the MARC field 856 to store the access URL, they inserted proxy server address information into the access URL for the title, which allowed authorized users to login to electronic resources from off campus, and used the canned phrase "Connect to online resource. Cal Poly Pomona users only" as the hyperlinked text that would display to users in the online catalog. Once a title was triggered for purchase, for a flat cataloging fee per record, YBP would deliver another bibliographic record along with purchase data for automated invoice ingestion and order record generation in the ILS. YBP constantly profiles new titles in its GOBI 3 ordering system, and new records that fit the approval profile for DDA discovery were to be provided on a weekly basis, via FTP through the ILS acquisitions interface (Millennium Acquisitions).

Other cataloging aspects were considered to aid BAS in maintaining discovery records. The existing batch load print monograph workflow had been adapted and modified for this purpose, 
whereby the discovery records were handled as little as possible, and the metadata accepted as-is in batch loads. Bibliographic and item records were created upon loading with a blank cataloging date field, and a custom fixed-field code indicating that the title was not to be counted as part of the department monthly acquisitions and holdings statistics. This custom code also served the purpose of excluding discovery records from appearing in monthly authority control/headings review reports, greatly reducing the amount of "noise" for the person who performed authorities maintenance work. After each discovery record load, the Millennium function "Global Update" was used to insert a local variable-length repeatable MARC field, flagging the record as "DDA Discovery" and providing an access point to collocate discovery records into a file for later review and batch editing.

When it came to the LCSH call numbers in the discovery records, CPP followed its local practice of "hiding" (suppressing) ebook call number information from the public's view in the online catalog. Librarians and users typically find virtual shelflisting (a visual display or list of books arranged by call number) to be a useful tool for resource discovery, but the Reference and Instruction team found that users were confused by the display of a call number in tandem with an electronic work, since they tended to associate call numbers with the physical location of an item in the building. Hiding call numbers also prevented discovery ebooks from appearing on "new title" and "featured title" lists in the online catalog, an automated feature which is driven by a record's call number and catalog date. It would be inappropriate to promote discovery ebooks as "new content," when that content is temporal and may never actually become property of the library. In order to hide the call numbers, the 050 and 090 call number MARC fields (which are indexed to display in the online catalog) were converted to 070 (also a call number field, but one that does not display), via Global Update. A simpler solution might have been to delete the call numbers from the discovery records altogether, but by retaining them in the 070 field, selectors could still analyze discovery record content by classification in the staff mode of the online catalog. 
A pilot seed amount of $\$ 30,000$ was identified and placed on deposit, out of which STLs, purchased titles, and cataloging fees were to be paid on a single accounting fund code. Allocating the starter funds into different fund codes by subject was briefly considered, but quickly discarded, as the Collection Development Team wanted to observe user behavior and resulting purchase activity primarily without imposed subject constraints or selector influence. It would be highly difficult to accurately predict which subject areas would experience heavier traffic than others, and which areas would have more outright purchases vs. STLs, and vice-versa. The wide range of publisher pricing in the approval profile also made expenditures difficult to project.

Great care was undertaken to ensure that discovery records would not be delivered that duplicated existing print and electronic holdings. To that effect, a master list of ISBNs was compiled to allow YBP to thoroughly comb through the initial DDA discovery record set and remove duplicates. YBP is CPP's primary book vendor, and thus this ISBN list would need infrequent updating moving forward, as they would already have the holdings data for most newly acquired titles. The first-time data dump of existing holdings' ISBN (020) MARC data into .csv format, and subsequent clean-up for use and DDA deduping by YBP, was quite the learning experience for the BAS Team. Staff could batch export bibliographic record data from the catalog using the Millennium ILS Data Exchange function, but the data was not homogenized in a form that was readable by YBP's systems. Multiple ISBNs would sometimes appear in the same row, with hyphens, commas, semicolons, or even additional text cluttering up the 10- and 13-digit numbers. Cells in Excel had to be formatted to general text, as numerical formatting sometimes resulted in the shortening of ISBNs into scientific notation. Staff were faced with the daunting task of manually cleaning up the spreadsheet and separating thousands upon thousands of ISBNs into separate cells in the spreadsheet.

Fortunately, the team was able to come up with a workaround, using built-in export tools and a specialized Excel add-on. The final procedure tooled the batch export to use a non-traditional character 
as a text delimiter for the .csv format - instead of using the standard comma or semicolon, the export file designated the circumflex ("up carrot") symbol to separate and delimit fields of text, as that character never coincidentally appeared in the ISBN fields of MARC records. Next, staff downloaded and utilized the free Excel add-on ASAP Utilities to automate the removal of commas, semicolons, and other erroneous text and characters from the ISBNs, with the tool's "advanced character remove or replace" function. A step-by-step procedure detailing process (with screenshots) was developed to aid staff in the future execution of this periodic task.

After the final contractual agreement had been signed, and a slight hiccup over garbled local metadata in the access URL (856) field had been ironed out, an initial DDA discovery record set of 2,195 titles was loaded into the online catalog and made available to the public's eyes on November 1, 2011. Despite the fact that the selectors decided to not advertise or promote the pilot in any way to library users, choosing to take a heuristic approach and keep the pilot's developments free from selector influence, the first STL was triggered the very next day on November $2^{\text {nd }}$.

STL activity, once triggered, was batched into groups and invoiced by YBP at regular intervals. A single purchase order record was created in Millennium Acquisitions and appended to a "dummy" bibliographic record to handle all STL activity and payments, allowing the Team to streamline payments and reduce excessive handling, in addition to being able to review STL expenditures at a glance. Finally, those selectors who were interested to see the exact breakdown of STL activity by title and cost could view the transactions within the ebrary admin website, or opt-in to a daily automatic email notification from ebrary that summarized all the DDA activity of the previous day.

\section{Evolving Workflows}

Within the first few weeks of the pilot, that first adaptation to the carefully planned out and agreed upon workflow occurred when the Collection Development Manager discovered abnormally high STL costs for several titles (one even topping $\$ 1,000$ dollars). Upon investigation, the Team learned that 
some titles exceeding the $\$ 200$ list price ceiling had slipped through the cracks and made it into the discovery record pool, owing to the fact that the DDA approval profile had been extracted from the print approval profile (which did include titles over $\$ 200$ ). The titles in the pool exceeding $\$ 200$ were immediately identified and pulled from both the pool and the online catalog. This incident resulted in more individuals opting-in to the DDA activity email generated by ebrary, as closer attention paid to DDA activity by more pairs of eyes would likely catch future abnormalities in a timely manner. A step was also added to the workflow, to review STL invoices for unusually high charges.

Next, the first triggered purchase activity elicited some minor squeaks from the wheels of the acquisitions process. For regular print purchasing activity through YBP, order requests are batched into a file at the end of business day and electronically submitted to the vendor. Upon processing by YBP, order metadata such as price, order type, purchase order number, and invoice number is generated and appended to a complete MARC bibliographic record for the title (when possible; sometimes minimal bibliographic data consisting of only the title and author is presented when a full record is not available). OCLC WorldCat Cataloging Partners (formerly PromptCat) generates a notification that the bibliographic and order records are ready to be downloaded via FTP, and the BAS member can then ingest both bibliographic records and invoice data ahead of receiving the physical item. This entire same practice was adopted for titles acquired via DDA purchase, with the exception that it was ebrary who batched and sent title purchase information to YBP on behalf of the library, and that ebooks were available to the user the moment they were triggered for purchase. However, it was soon found that this linear progression of acquisitions tasks did not work for the DDA workflow: the availability of YBP invoices for purchased DDA, and the OCLC notification that bibliographic records were ready for pickup, were often out-of-sync. Most often, the OCLC notification would arrive days or even a week ahead of the YBP invoices. After some initial confusion, BAS adapted the workflow to await invoices from YBP before 
beginning to process DDA purchases, essentially disregarding the usual OCLC record notifications that were flagged with the DDA account number.

Another challenge to the workflow arose in those early months when the presence of discovery records that duplicated existing holdings was uncovered. And, upon investigation, it was further revealed that the duplicates occurred through no one's particular fault or oversight. The duplicates are actually the fault of the essential nature (and drawback) of holding local copies of MARC records in an ILS system, in that there are multiple silos and knowledge bases of data to maintain (the ILS, YBP, other vendors, and OCLC). The data amongst the different knowledge bases may not be in sync, and have no way to communicate with one another without mediation by a human being (usually a cataloger who marks holdings in each data silo). Thus, YBP had no way of knowing that the ProQuest SafariTech rotating ebook collection, owned centrally by the California State Library Consortium (SDLC), had added titles that duplicated Cal Poly Pomona DDA discovery records. Similarly, other subscription ebook packages leased by Cal Poly Pomona grow and acquire publishers and titles represented in the DDA pool, particularly the Wiley imprint of IEEE Xplore.

In order to reflect the addition and departure of titles from the SafariTech package, BAS refreshes its bibliographic record set in the online catalog on a monthly basis. Other ebook packages are updated in the catalog as changes occur and notification is received. Some libraries utilize the ILS batch ingest template during this process to detect title duplicates upon load - the system will reject any incoming records that duplicate records already found within the catalog. However, the local BAS template for batch loading ebook records is purposely designed to not overlay existing records in the catalog, as BAS cataloging policy is to create a separate vendor-neutral record for each provider of a given ebook title. Even if overlay were activated for these records, a duplicate would not have been detected - for at the time of loading the discovery records, no duplicates yet existed in the catalog. It was only when titles rotated in to SafariTech and other ebook packages that records for now owned 
content were loaded that duplicated records for potential to own titles. We are hardly the first library to experience this maddening phenomenon, as the difficulty of identifying and merging duplicate ebook records via human cataloger comparison is outlined by the University of Houston Libraries in their discussion of mass management of catalog records. ${ }^{8}$

The duplicate records went unnoticed for some time, and it was only in carrying out the triggered purchase cataloging workflow that they were finally discovered. Upon receiving invoice notification from YBP, the cataloger used Millennium Acquisitions to upload the OCLC-delivered records and purchase data into the catalog. A cataloging date was applied, the item statistics fixed field was encoded to include the record in holdings counts, and a local bibliographic field was added to indicate that the title was a "DDA Purchase" (serving as an access point for record collocation and recall). The cataloger then searched for and deleted the corresponding discovery record from the catalog.

In this instance, when the cataloger went to search for and delete the discovery record for a purchased title, her title search recalled more than the anticipated two records for the title (one discovery, one purchase). In fact, there were some instances where as many as four records were recalled, as the title had been added to more than one ebook content provider (e.g. one discovery, one purchase, one IEEE Xplore, and one SafariTech). Comparison of the creation dates for these records revealed that the duplicative content had been loaded into the catalog after the ingest of DDA discovery records. And further digging into the dates of STL that triggered the purchase revealed that STLs usually occurred prior to the subscription content being added to the catalog. Thus, STL funds were expended in addition to owning the content from another provider, through no one individual's fault. In this instance, the workflows for both DDA discovery ingest and regular ebook subscription maintenance were adjusted to include a step for reviewing the post-batch load report, to identify possible DDA

\footnotetext{
${ }^{8}$ Annie Wu and Anne M. Mitchell, "Mass Management of E-Book Catalog Records: Approaches, Challenges, and Solutions," Library Resources and Technical Services 54, no. 3 (2010): 164-173. http://dx.doi.org/10.3359/oz1003138
} 
discovery duplicates and delete the discovery record if necessary. The title was then removed from DDA on the ebrary admin website, to ensure it would not inadvertently return if for some reason all the DDA records were to be reloaded.

This practice, while successful in identifying and removing duplicates, remains to this day an inefficient means of managing monthly loads of thousands of batch titles as we add new content providers to the ebook mix (most notably, EbscoHost and JSTOR titles). However, there is hope for the future. The California State University Library system has adopted and is in the process of implementing Ex Libris' Alma as its unified library management system (ULMS). Alma's architecture is built on the Oracle programming language, meaning programmers can develop SQL queries to expose data from the catalog that was previously inaccessible and hidden on the proprietary III Millennium platform. With the entire skill set and talents of all CSU librarians and staff brought to bear, it is my hope that a SQL query will be developed to automatically and regularly seek and report on title duplicates in the unified catalog, saving staff the work of manually searching for duplicates across hundreds and hundreds of titles. In the meantime, the problem of STLs occurring for titles that might someday, maybe, become owned content via one of the subscribed ebook packages seems unavoidable, and a terrible waste of precious resources.

More recently, challenges arose in the form of an ongoing SDLC Pilot DDA project across multiple ebook platforms, resulting in purchased content owned by the consortium and accessible to all member libraries. Care was taken to encode the CSU DDA records separately from the local DDA records, as the CSU records are deleted after the pilot money for each platform has been depleted. Also, the CSU project coordinator worked extensively with the content providers to ensure that content 
owned by the consortium would not unduly duplicate holdings of the 23 individual libraries represented in the system. ${ }^{9}$

Finally, the presence of DDA discovery records in the public catalog and discovery layer has led to a change in selector behavior for at least two members of the Collection Development Team, which then revealed another DDA snag. One selector had refrained from purchasing a particular title earlier in 2012, because she noticed that it had been added to our DDA pool, and so she wished to sit back and see whether the title would be purchased via DDA/user behavior. However, when 2013 rolled around and no STL activity had occurred, she decided to intervene and initiate a purchase of the work after all. To her dismay, she found that the ebook URL from the discovery record led to a screen stating that the title was no longer available for DDA. The BAS Team investigated, and found that several hundred titles had seemingly dropped out of the DDA pool with no notification. YBP was immediately contacted for assistance, and began work with ebrary to discover a solution. Even more mysteriously, some of the affected titles sporadically "came back" into the pool after investigations were begun. The exact cause of the incongruity between YBP and ebrary data was never discovered, but with above-and-beyond assistance from YBP, the BAS Team was able to do a complete dump and reload of discovery records to wipe the slate, and eliminate the hundreds of "dead" discovery links in the public catalog.

\section{Conclusion}

Ultimately, the DDA Pilot at Cal Poly Pomona has been a resounding success. The Pilot was extended past its initial one-year mark, as a significant number of DDA funds had yet to be expended, and it will likely continue to run until the deposit account is exhausted. As of November 2015, over 29,600 discovery records have been loaded, and 518 titles have been purchased. The DDA deposit account is an attractive way to protect funds that are subject to the wild fluctuations of state support,

\footnotetext{
9 Jodi Shepherd and Marc Langston, "Shared patron driven acquisition of E-books in the California State University Library Consortium," Library Collections, Acquisitions, \& Technical Services 37, no. 1-2 (2013): 34-41. http://dx.doi.org/10.1080/14649055.2013.10766345
} 
and the Collection Development Team feels that DDA has overall enhanced the selection of relevant resources for library users. DDA is an exceedingly worthwhile endeavor, and its success will only continue to grow with the continued collaborative, responsive, and adaptive efforts of Collection Development and the BAS Team.

Like so many publicly-funded libraries, the Cal Poly Pomona University Library has experienced over a decade of workforce attrition due to funding crises and subsequent hiring freezes. The BAS Team grows ever-leaner, and the concentration of print and electronic monograph record management skills has been distilled into a few individuals who must constantly adapt to pre-existing and unforeseen challenges. As such, the DDA acquisition workflow remains fluid and changeable, constantly evolving to leverage the power of point-of-need acquisitions against the obstacles facing the mass management of records in a static local catalog.

\section{Bibliography}

Allison, Dee Ann. The Patron-Driven Library: A Practical Guide for Managing Collections and Services in the Digital Age. Oxford: Chandos Publishing, 2013.

Anderson, Rick. "Collections 2021: The Future of the Library Collection Is Not a Collection," Serials 24, no. 3 (2011): 211-215. doi: 10.1629/24211.

Emery, Jill, and Bonnie Parks. "The Demand Driven Acquisitions Pilot by the Orbis Casacade Alliance: An Interview with Members of the Demand driven Acquisitions Implementation Team." Serials Review 38 (2012): 132-136. doi: 10.1080/00987913.2012.10765440.

Pool, Rebecca. "Big-Deal Packages Squeeze Recession-Hit Libraries." Research Information 48 (2010). http://www.researchinformation.info/features/feature.php?feature_id=269.

Schroeder, Rebecca, and Tom Wright. "Electronic Books: A Call for Effective Business Models." New Library World 112, no. 5/6 (2011): 215/221. doi: 10.1108/03074801111136257. 
Serial Price Projections for 2015. Ipswich, MA: Ebsco, 2014. Accessed September 1, 2015. http://www.ebscohost.com/promoMaterials/Serials_Price_Projections_for_2015.pdf.

Shepherd, Jodi, and Marc Langston. "Shared patron driven acquisition of E-books in the California State University Library Consortium," Library Collections, Acquisitions, \& Technical Services 37, no. 1-2 (2013): 34-41. doi: 10.1080/14649055.2013.10766345.

Way, Doug and Julie Garrison. "Financial Implications of Demand-Driven Acquisitions: A Case Study of the Value of Short-Term Loans." In Patron Driven Acquisitions: History and Best Practices, edited by David A. Swords, 137-156. Berlin: De Gruyter Saur, 2011.

Wu, Annie and Anne M. Mitchell. “Mass Management of E-Book Catalog Records: Approaches, Challenges, and Solutions," Library Resources and Technical Services 54, no. 3 (2010): 164-173. doi: 10.3359/oz1003138. 